\title{
Sub-bandgap photoconductivity in $\mathrm{ZnO}$ epilayers and extraction of trap density spectra
}

\author{
K Moazzami $^{1}$, T E Murphy ${ }^{1}$, J D Phillips ${ }^{1}$, M C-K Cheung ${ }^{2}$ and \\ A N Cartwright ${ }^{2}$ \\ ${ }^{1}$ Department of Electrical Engineering and Computer Science, The University of Michigan, \\ Ann Arbor, MI 48109-2122, USA \\ ${ }^{2}$ Department of Electrical Engineering, University at Buffalo, The State University of \\ New York, Buffalo, NY 14260, USA \\ E-mail: kmoazzam@umich.edu
}

Received 2 December 2005, in final form 13 January 2006

Published 6 April 2006

Online at stacks.iop.org/SST/21/717

\begin{abstract}
Photoconductivity is observed in $\mathrm{ZnO}$ epilayers due to photoexcitation in the visible spectral region of $400-700 \mathrm{~nm}$, below the ZnO bandgap energy of $3.4 \mathrm{eV}$. Photoconductive transients due to visible photoexcitation have time constants in the order of minutes. Treatment of the $\mathrm{ZnO}$ surface with $\mathrm{SiO}_{2}$ passivation layers results in a significant reduction in the photoconductive signal and photoconductive time constant. The photoconductive response is attributed to hole traps in $\mathrm{ZnO}$, where a rate equation model is presented to describe the photoconductive characteristics. A method of extracting the hole trap density spectrum is presented on the basis of the rate equation model and assumptions for hole capture lifetime and carrier recombination lifetime that are validated by experimental time-resolved photoluminescence measurements of the material under study. Traps are found to be distributed near $0.75 \mathrm{eV}$ and $0.9 \mathrm{eV}$ from the valence band edge for $\mathrm{SiO}_{2}$ passivated and unpassivated $\mathrm{ZnO}$ epilayers, respectively.
\end{abstract}

\section{Introduction}

$\mathrm{ZnO}$ and related materials are attractive for optoelectronic devices operating in the ultraviolet (UV) due to their excellent optical properties and availability of native substrates for lattice-matched epitaxy. The presence of a high number of trap and defect levels in both the bulk and the surface of the material is a major impediment to the realization of p-type material and junction devices. These defects will also strongly influence carrier dynamics in the materials, and are deleterious for device applications. Moreover, persistent photoconductivity and spectral response corresponding to energies lower than the bandgap energy of the material are problematic characteristics that occur as a result of these defects. These effects have been observed not only in $\mathrm{ZnO}$ [1-5] but also in other wide bandgap semiconductors such as $\mathrm{GaN}[6,7]$. In particular, rejection of response to visible and longer wavelength radiation is a primary goal for 'solar-blind' and other UV detector and imaging applications. Photoluminescence spectra further provide evidence of these deep levels, where 'green' emission is typically observed in $\mathrm{ZnO}$ with a peak corresponding to $1 \mathrm{eV}$ below the bandgap energy [8-14]. A developed understanding of the energetic and spatial distributions of defects in $\mathrm{ZnO}$ material that are the source of observed photoconductivity and long wavelength response is critical for device applications. Previously, two methods for the extraction of trap density from photoconductivity transients based on Laplace transform have been suggested $[4,5,15]$. The method presented here is similar to these methods since it also involves a transformation from time domain to spectral or energy domain. However, since our method is a constrained optimization method it will avoid non-real trap distributions involving negative trap densities at some energies whereas the methods previously suggested can result in such distributions. In this work, carrier trapping phenomena associated with these defects are studied through photoconductive transients and time-resolved photoluminescence measurements. The energetic distribution 
of these defects is resolved using a rate equation model for the photoconductive process, and the influence of surface defects on photoconductive transients is studied through experiments on passivated and unpassivated samples.

The assumptions necessary for the extraction of trap density spectrum from the photoconductivity transients are discussed in detail in this work. Since the technique described here for the extraction of the trap density spectrum is based on pure numerical optimization, the true trap density spectrum can be extracted from photoconductivity transients given the basic underlying assumptions made are satisfied. In this study we have limited ourselves to persistent photoconductivity measurements under constant white light illumination and the slow photoconductivity transients resulting before and after illumination. Thus we are specifically probing trap densities with large energies (energy separation of $0.6-1 \mathrm{eV}$ from the band edge). It has been observed that under excitation with pulsed laser source with short pulses (pulse widths of about $10 \mathrm{~ns}$ ) and energies larger than the bandgap of $\mathrm{ZnO}$, samples exhibit photoconductivity transients with much smaller time constants (in $\mu$ s and ms range) $[16,17]$.

\section{Experiment}

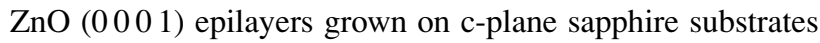
by plasma-assisted molecular beam epitaxy were studied in this work, with details of the growth process and material properties described previously $[16,18]$. Three samples were studied with n-type conductivity, thickness between 0.5 and $1.5 \mu \mathrm{m}$, electron concentration of $6 \times 10^{17}-3 \times$ $10^{18} \mathrm{~cm}^{-3}$ and electron mobility of $8-40 \mathrm{~cm}^{2} \mathrm{~V}^{-1} \mathrm{~s}^{-1}$. Following growth, test structures were fabricated for photoconductivity measurements through the definition of $0.6 \times 1 \mathrm{~mm} \mathrm{ZnO}$ bars using photolithography and wet chemical etching. Ohmic contacts were deposited as described previously [19]. $\mathrm{SiO}_{2}$ layers were deposited by chemical vapour deposition on photoconductor test structures from each sample to study the effect of passivation. Prior to passivation, the surface of samples was cleaned in $30 \% \mathrm{H}_{2} \mathrm{O}_{2}$ for $15 \mathrm{~min}$ as suggested by Shimizu et al [20].

Time-resolved photoluminescence (TRPL) was studied with a 200 fs pulsed laser source and streaked camera equipped monochromator. The laser pulses for excitation were generated by a regenerative amplifier (Coherent: RegA) that was seeded by a Ti:sapphire laser. The wavelength of the laser source was $800 \mathrm{~nm}$; therefore two $\mathrm{BBO}\left(\mathrm{BaB}_{2} \mathrm{O}_{4}\right)$ crystals were used to frequency triple the pulses to $266 \mathrm{~nm}$ for excitation. The frequency-tripled pulses were directed and focused on the sample by conventional optics, with an incident intensity in the order of $1 \mathrm{~mJ} \mathrm{~cm}^{-2}$ per pulse. Part of the resulting luminescence was directed into the streak camera equipped monochromator for spectral and temporal analysis. For the study of photoluminescence (PL), an $\mathrm{Ar}^{+}$ laser tuned to $351 \mathrm{~nm}$ was used for exciting the sample, and the same monochromator was used. In this case, the streak camera was operating in focus mode, where its time-resolving capability was turned off. Photocurrent transients of the $\mathrm{ZnO}$ photoconductors were studied under a constant bias of $2 \mathrm{~V}$. An incandescent lamp with an output spectrum $>400 \mathrm{~nm}$ was used with an output power of approximately $400 \mathrm{~W} \mathrm{~cm}^{-2}$. The

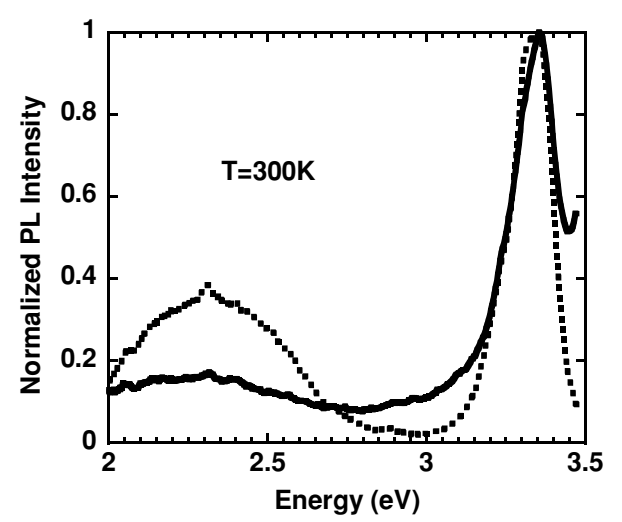

Figure 1. Room temperature photoluminescence spectra indicating the band edge and defect emission (green band) for two different samples of $\mathrm{ZnO}$ material under study.

samples were left in dark for at least $10 \mathrm{~h}$ before the start of measurement to reach dark equilibrium. Both the rise and fall of the photoconductive response were recorded for an hour before and after the incident excitation was turned off.

\section{Results}

Photoluminescence spectra of the $\mathrm{ZnO}$ samples show a sharp band edge peak and a broad deep level peak near $2.35 \mathrm{eV}$ (green band) as shown in figure 1 for two different samples featuring different defect level to band edge peak ratios representing different capture lifetimes or density of defect levels. Deep recombination centres close to the valence band edge present in the material are believed to be the origin of these luminescence peaks $[8,9,12,14]$. TRPL measurements provide a means of comparing the relative magnitudes of the carrier recombination lifetime and capture lifetime into deep levels, with results shown in figures $2(a)$ and $(b)$. The recombination time for minority carriers, including band-to-band recombination through both exciton states and recombination centres can be approximated by the decay time constant of the TRPL band edge emission shown in figure $2(a)$, and is in the order of 100 ps. The PL decay from the deep levels is significantly longer than that of the band edge, in the order of $20 \mathrm{~ns}$, as indicated by the TRPL data shown in figure $2(b)$. The TRPL lifetime for these defect states is determined both by carrier capture into the defect states and the radiative recombination lifetime. Radiative recombination time for the defect level can be extracted from the decay portion of the TRPL measurements. The TRPL decay time for defect emission is longer than the rise time of the defect level TRPL lifetime, where the total defect level TRPL lifetime is a combination of carrier capture and recombination associated with the defect states. We may approximate the capture time into the defect levels by the rise time of the defect level TRPL, approximately $20 \mathrm{~ns}$. The TRPL decay corresponding to band edge emission indicates a band edge recombination time on the order of $100 \mathrm{ps}$. We can readily conclude that the capture time into these defect levels is orders of magnitude longer than band edge recombination lifetime.

The photoconductive transient response due to visible illumination shows a decay time in the order of $80 \mathrm{~min}$, as 


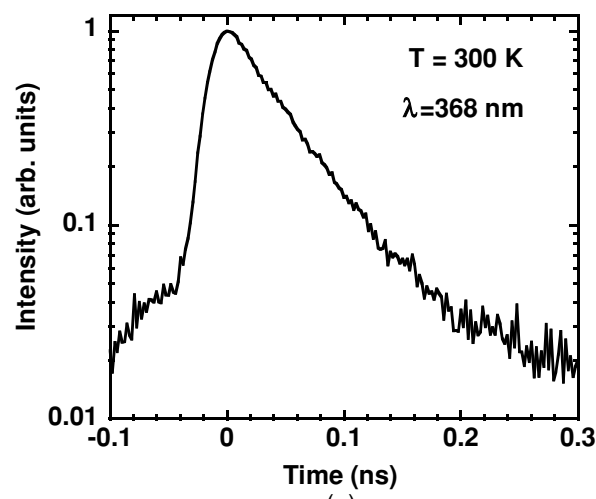

(a)

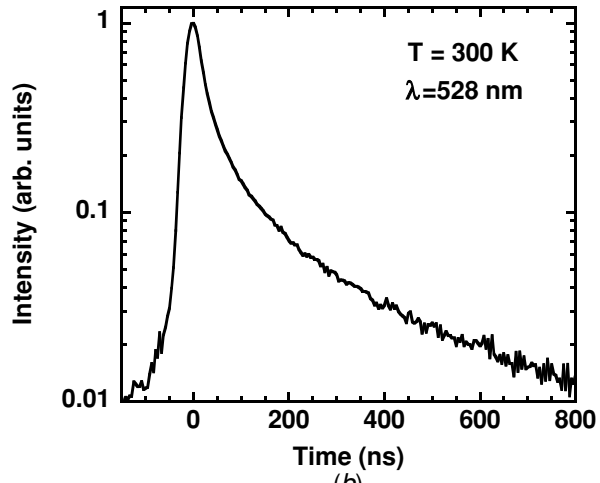

(b)

Figure 2. Room temperature time-resolved photoluminescence intensity for both (a) band edge (near $368 \mathrm{~nm}$ ) and (b) defect level (near $528 \mathrm{~nm}$ ) emissions.

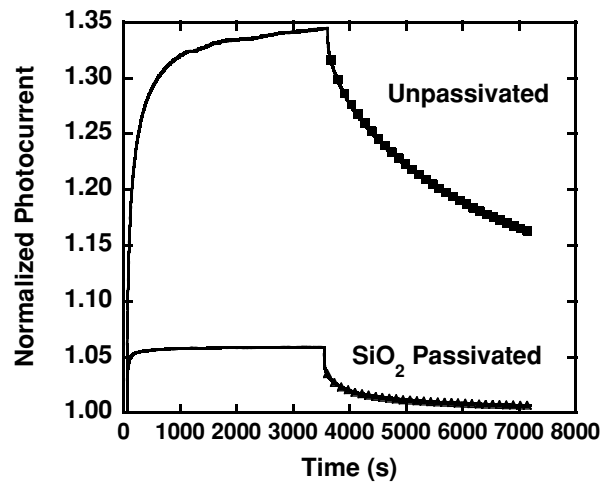

Figure 3. Photoconductive transients under visible excitation for a $\mathrm{ZnO}$ epilayer with and without passivation, and the corresponding fit (shown by symbols) using the rate equation model.

indicated in figure 3. A significant reduction in the decay time is observed for samples passivated with $\mathrm{SiO}_{2}$, where the time constant is reduced to approximately $6 \mathrm{~min}$. All the three samples studied demonstrated similar behaviour, including a clear reduction in the photocurrent and decay time constant upon passivation with $\mathrm{SiO}_{2}$. It has been previously observed that oxygen physically adsorbed on the surface of $\mathrm{ZnO}$ plays an important role in the photoconductivity transients observed in $\mathrm{ZnO}$ [5]. This can possibly explain the change in the photoconductivity transients that we observed after passivation of the surface of the sample when ambient oxygen is prevented from interacting with the surface of $\mathrm{ZnO}$.

\section{Discussion}

A proposed model for processes that occur during the photoluminescence measurements and a proposed model for the photoconductive behaviour are depicted in figures 4(a) and (b) respectively and may be summarized as follows. During the photoluminescence and time-resolved photoluminescence using the UV illumination, as depicted in figure 4(a), electron-hole pairs are generated corresponding to band-toband transitions. After the illumination is turned off, the excess holes either recombine with the excess electrons created in the conduction band through the recombination centres present in the material and the exciton annihilation process or get captured into deep recombination centres close to the valence band edge or hole trap levels and either recombine with electrons in the conduction band or stay trapped in the trap level depending on whether the defect level is a recombination centre or a trap. The recombination process can happen either radiatively or non-radiatively for both the band edge recombination and trap level to conduction band recombination. The radiative portion of the recombination can be traced by looking at the photoluminescence spectra and time-resolved photoluminescence transients for the band edge and defect level emissions.

In the case of photoconductivity transients due to visible excitation as shown in figure $4(b)$, electron-hole pairs are generated upon visible excitation, corresponding to optical transitions between defect states and the band edge. The slow photoconductive decay observed in these experiments may be attributed to the capture of holes in deep trap levels, and subsequent hole emission and carrier recombination. The time constant of the transient decay observed is governed by the depth of these traps and can be very long. Point defects behaving as hole (or electron) traps, in contrast to defect centres, exhibit slow electron-hole recombination where trapped carriers are typically re-emitted into the valence (or conduction) band. In many cases, hole trap characteristics can be attributed to a negative charge when the trap is filled with a hole, resulting in a repulsive charge to electrons and small electron capture cross section. This type of behaviour has been observed for a deep hole trap in GaN [21]. Deep hole traps at $0.8-0.9 \mathrm{eV}$ with respect to the valence band edge have been reported for $\mathrm{GaN}$ (a similar wide bandgap semiconductor material) by several authors [21-26]. Most recently, Salis et al [27] have attributed persistent photoconductivity in GaN to traps in the material with a small electron-hole recombination rate. The excess holes generated by visible illumination remain captured in the hole traps until they are thermally emitted into the valence band. Electron traps will be nearly filled and will not contribute to the transient photoconductivity observed because of the high n-type carrier concentration of the $\mathrm{ZnO}$ material and resulting electron quasi-Fermi level near the conduction band. Under visible excitation for a long time, a steady-state photocurrent will be achieved upon saturation of hole traps. If the illumination time is not long enough compared to the time constant of the turn-on of the transient photoconductivity, a quasi-equilibrium is reached where the hole distribution function over the traps depends on the intensity and period of illumination. In general it takes less illumination time to saturate the hole traps in passivated 


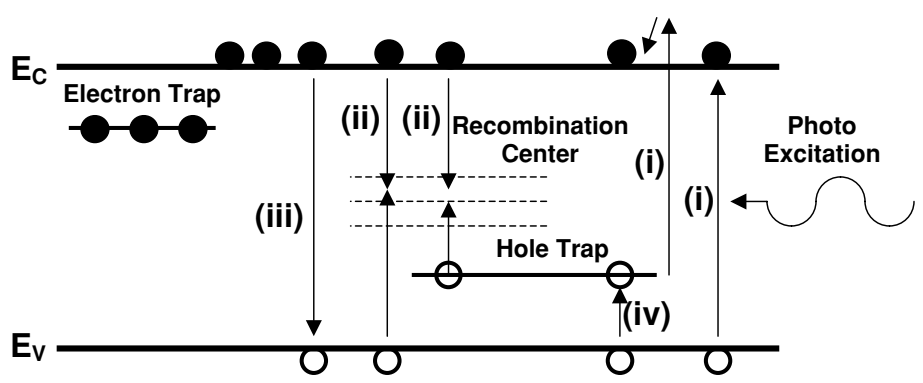

(a)

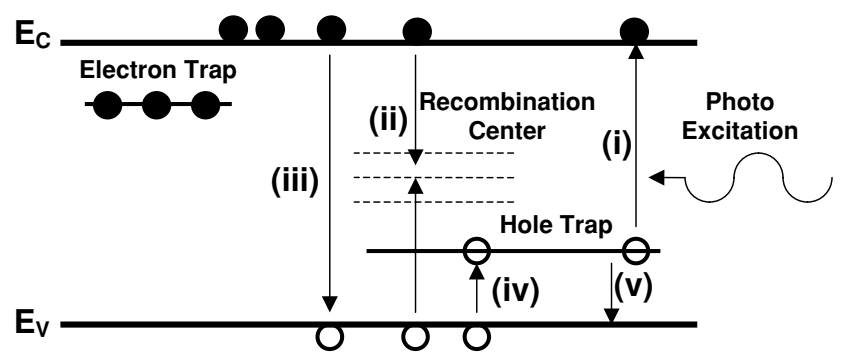

(b)

Figure 4. Schematic illustration of the proposed model for significant (i) electron-hole pair photogeneration, (ii) recombination through recombination centres, (iii) band-to-band (exciton) recombination, (iv) hole capture and (v) hole emission processes that occur during (a) photoluminescence and $(b)$ photoconductivity experiments.

samples because of shallower traps present in the material. The increase in photoconductivity relative to dark conditions will result from an increase in electron concentration in the conduction band corresponding to approximately the number of filled hole traps. After the excitation is turned off, the photocurrent will change according to the excess electron concentration which will deplete upon recombination with holes emitted from hole traps into the valence band. Holes in traps will thermally emit and either recombine with the excess electrons in the conduction band or get recaptured into the hole trap levels. Rate equations that represent these photoconductive processes corresponding to visible excitation are as follows:

$$
\begin{gathered}
\frac{\mathrm{d} n}{\mathrm{~d} t}=\Phi \sigma_{\mathrm{p}, \text { light }}\left(N_{\mathrm{T}}-N\right)-\frac{p}{\tau_{\text {recombination }}}, \\
\frac{\mathrm{d} N}{\mathrm{~d} t}=\Phi \sigma_{\mathrm{p}, \text { light }}\left(N_{\mathrm{T}}-N\right)-N s \mathrm{e}^{-\frac{E_{\mathrm{p}}}{K T}}+\frac{p}{\tau_{\text {capture }}}, \\
\frac{\mathrm{d} p}{\mathrm{~d} t}=N s \mathrm{e}^{-\frac{E_{\mathrm{p}}}{K T}}-\frac{p}{\tau_{\text {capture }}}-\frac{p}{\tau_{\text {recombination }}},
\end{gathered}
$$

where the attempt to escape frequency for holes $s$ is given as

$$
s=\sigma_{\mathrm{p}} v_{\text {thermal, } \mathrm{p}} N_{\mathrm{v}},
$$

where $n$ and $p$ are the conduction band electrons and valence band holes, $N_{\mathrm{T}}$ is the total density of hole traps, $N$ is the number of filled hole traps, $\Phi$ is the incident light flux, $\sigma_{\mathrm{p} \text {,light }}$ is the capture cross section of the hole traps for incident light, $N_{\mathrm{v}}$ is the valence band effective density of states and $E_{\mathrm{p}}$ is the energy separation between the hole trap level and the edge of valence band. If the capture lifetime is much larger than the band edge recombination lifetime, holes emitted from traps will primarily recombine rather than being recaptured into another hole trap. The process of hole capture may then be neglected.
As a result, excess conduction band electrons will decay with a time constant governed by the thermal emission from hole traps resulting in the simplified relationship between hole traps and conduction band electrons given by

$$
\int N_{\mathrm{T}}\left(E_{\mathrm{i}}\right) \exp \left(-s \mathrm{e}^{-\frac{E_{\mathrm{i}}}{K T}} t\right) \mathrm{d} E=\Delta n(t) .
$$

To better understand how equation (5) is extracted from equations (1)-(4), we note that the recombination process is rather instantaneous compared to the emission process and the recombination time (in the order of ns) is much shorter than the time scales under consideration for the transient photoconductivity observed (in the order of minutes) and hence we have

$$
\left|\frac{\mathrm{d} p}{\mathrm{~d} t}\right| \ll\left|\frac{p}{\tau_{\text {recombination }}}\right| .
$$

We have also assumed that the recombination time is much shorter than the capture time of the holes into defect levels, so we have

$$
\frac{p}{\tau_{\text {capture }}}+\frac{p}{\tau_{\text {recombination }}} \cong \frac{p}{\tau_{\text {recombination }}},
$$

resulting in a simplified version of equation (3):

$$
\frac{\mathrm{d} p}{\mathrm{~d} t}=N s \mathrm{e}^{-\frac{E_{\mathrm{p}}}{K T}}-\frac{p}{\tau_{\text {recombination }}} .
$$

Equation (6) can also be verified by noting the fact that the number of holes present in the valence band is very small compared to the number of hole-filled hole traps contributing to the emission process resulting in

$$
\left|\frac{\mathrm{d} p}{\mathrm{~d} t}\right| \ll\left|N s \mathrm{e}^{-\frac{E_{\mathrm{p}}}{K T}}\right| .
$$

Using either of the equations (6) or (9) we can conclude that

$$
\frac{p}{\tau_{\text {recombination }}}=N s \mathrm{e}^{-\frac{E_{\mathrm{p}}}{K T}}-\frac{\mathrm{d} p}{\mathrm{~d} t} \cong N s \mathrm{e}^{-\frac{E_{\mathrm{p}}}{K T}},
$$




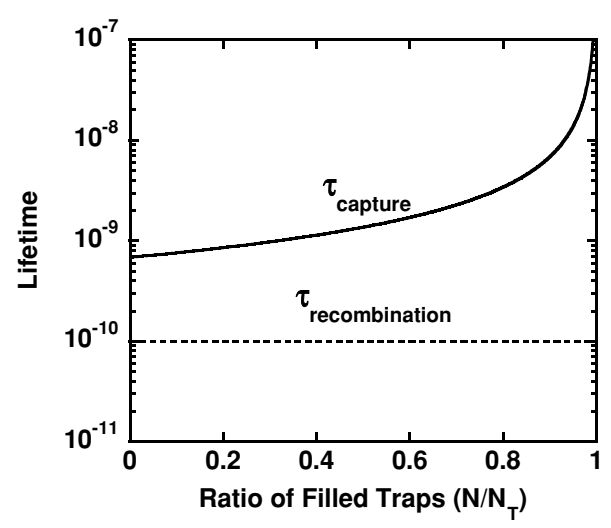

Figure 5. Comparison of typical capture and recombination lifetimes as a function of ratio of filled hole traps.

which upon substitution into equation (1) and considering only the decay portion of the process when the excitation is turned off will have

$$
\frac{\mathrm{d} n}{\mathrm{~d} t}=-\frac{p}{\tau_{\text {recombination }}}=-N s \mathrm{e}^{-\frac{E_{\mathrm{p}}}{K T}} .
$$

Knowing that the transient decay process observed is a result of emission from trap levels of various energies we can separate equation (11) into different energies and write

$$
\frac{\mathrm{d} n(E)}{\mathrm{d} t}=-N(E) s \mathrm{e}^{-\frac{E_{\mathrm{p}}}{K T}}
$$

resulting in

$$
n(E)=N_{\mathrm{T}}(E) \exp \left(-s \mathrm{e}^{-\frac{E_{\mathrm{p}}}{K T}} t\right),
$$

where we have replaced $N_{\mathrm{T}}(E)$ for the initial concentration of excess electrons by the assumption that the hole traps are completely saturated when the illumination is turned off and the decay is observed. This equation can in turn be integrated over all the energies to yield equation (5).

To satisfy the condition of longer hole capture lifetime than electron-hole recombination lifetime, it is sufficient to satisfy it at dark equilibrium when hole traps are empty and there are no excess electrons in the conduction band. This dark condition represents the condition of the shortest hole capture lifetime. The relationship between the capture lifetime and the number of filled hole traps is given as

$$
\tau_{\text {capture } \mathrm{p}}=\frac{1}{\sigma_{\mathrm{p}} v_{\text {thermal }, \mathrm{p}}\left(N_{\mathrm{T}}-N\right)},
$$

and the band edge recombination time is independent of the number of filled hole traps and can be assumed to be on the order of $100 \mathrm{ps}$ as experimentally observed in figure 2 .

Assuming that the trap concentration is less than the n-type carrier concentration of approximately $10^{17} \mathrm{~cm}^{-3}$ for the MBE grown samples under study, the hole capture cross section would have to be $\sigma_{\mathrm{p}}<10^{-15} \mathrm{~cm}^{2}$ for a capture lifetime of $\tau_{\text {capture,p }}>1 \mathrm{~ns}$, which is a typical value for trap cross section. TRPL results further support this conclusion, where the band edge recombination lifetime is in the order of $100 \mathrm{ps}$ and is much smaller than the minimum capture lifetime of $\tau_{\text {capture,p }}>$ $1 \mathrm{~ns}$ given above and capture lifetime value of approximately 20 ns suggested by TRPL results. Figure 5 shows a comparison between the capture lifetime and the band edge recombination lifetime with $\tau_{\text {recombination }}=100 \mathrm{ps}, \sigma_{\mathrm{p}}=10^{-15} \mathrm{~cm}^{2}, N_{\mathrm{T}}=$ $10^{17} \mathrm{~cm}^{-3}, m_{\mathrm{h}}=0.64 m_{0}$ and $v_{\text {thermal, }}=1.46 \times 10^{7} \mathrm{~cm}^{2} \mathrm{~s}^{-1}$.

The trap density spectra may then be extracted from photoconductive decay transients according to equation (5) provided the argument that recapture of holes into traps may be neglected. This trap density extraction is performed by minimization of the overall error in the prediction of the transient current searching on all the trap density functions having non-negative values for trap density based on the transient photocurrent and trap density relationship defined by equation (5). Since this is a numerical optimization, the resulting trap density function can assume any shape as long as the values of trap density at each energy are nonnegative. Increasing the energetic resolution of the extracted trap density function will result in increased simulation time. The resolutions we achieved were better than $5 \mathrm{meV}$ with a reasonable convergence time. The carrier density versus time corresponding to this extraction shows excellent agreement with the measured photoconductive transient data, as shown in figure 3 where markers represent the predicted photocurrent transients resulting from the optimum trap density function and solid lines represent the measured photocurrent transients. The value of attempt to escape frequency for holes was assumed to be equal to $s=1 \times 10^{11} \mathrm{~Hz}$, corresponding to a hole trap in $\mathrm{ZnO}$ with a capture cross section of $\sigma_{\mathrm{p}}=5.34 \times 10^{-16} \mathrm{~cm}^{2}$, throughout all these calculations. Assuming the hole mass in $\mathrm{ZnO}$ to be equal to $m_{\mathrm{h}}=0.64 m_{0}$, the value of attempt to escape frequency of holes in $\mathrm{ZnO}$ is found to vary between $1.87 \times$ $10^{9}$ and $1.87 \times 10^{11} \mathrm{~Hz}$ for typical capture cross sections of $10^{-17}$ to $10^{-15} \mathrm{~cm}^{2}$. Assuming an order of magnitude smaller value for this parameter results in an approximately $60 \mathrm{meV}$ shift in the extracted trap spectrum. This energy value is relatively small given our results, indicating that the trap energy spectrum has a weak dependence on the value assumed for $s$. The photocurrent magnitude and transient time constant have a direct relation with the trap density and energy separation from the band edge, respectively, in this analysis.

The resulting trap densities extracted show a peaked distribution for the three samples under study, as shown in figure 6. All the unpassivated samples show a peaked response near $0.9 \mathrm{eV}$, where a clear decrease in trap density and shift to a peak near $0.8 \mathrm{eV}$ is observed after passivation. The extracted functions are the optimum solutions that satisfy equation (5) with a minimum overall error over the whole transient. Any change in the shape or magnitude of this function can result in large deviation of the transient from the experimentally measured curve. The artificial peaks, dips and kinks observed in the extracted trap density spectrum for the unpassivated sample are primarily because of the fact that the transient photocurrent of the unpassivated samples was not fully saturated after $1 \mathrm{~h}$ under illumination. Noise present in the transient photocurrent can also result in such features. To extract a more correct representation of the trap density spectrum, the transient photoconductivity should be measured for several hours under illumination so that the transient photocurrent is completely saturated. Measurement of the transient photocurrent for several hours after illumination until the transient is completely settled will also result in a more accurate trap density spectrum and also extension of the trap density spectrum to probe deeper traps with larger 


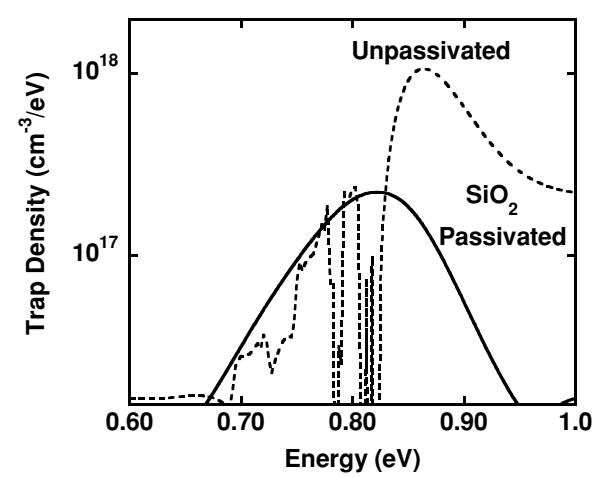

(a)

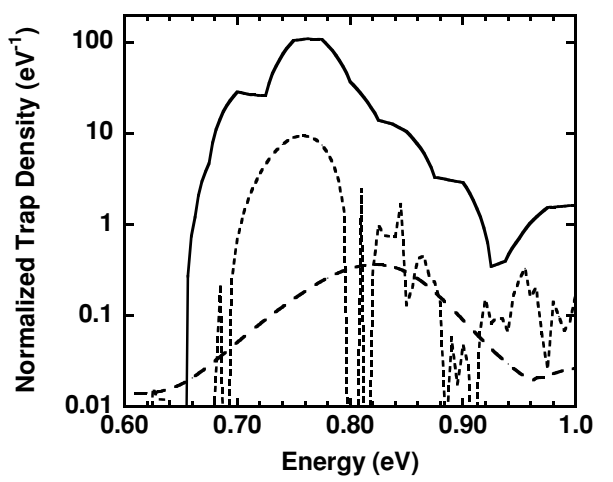

(b)

Figure 6. Extracted trap density spectra for (a) a $\mathrm{ZnO}$ sample with and without $\mathrm{SiO}_{2}$ passivation and $(b)$ three different passivated samples normalized to the background carrier concentration of each sample.

separation from the band edge energies as well. The reduction in the total trap density as a result of passivation indicates that the transient photoconductivity observed is dominated by deep trap levels present on the surface of the material. The photoconductive response for passivated samples is likely determined by traps that are either distributed in the epilayer or present at the $\mathrm{ZnO} / \mathrm{Al}_{2} \mathrm{O}_{3}$ interface. Unlike the case of defect levels responsible for the defect level emission, the captured hole traps into these levels do not recombine after getting captured and will only get emitted to the valence band after thermal excitation. The extracted trap energy spectrum peaked near $0.8 \mathrm{eV}$ is close to the green band emission centred at $2.35 \mathrm{eV}$ and $1.0 \mathrm{eV}$ below the band edge. It is important to note that the deep levels responsible for the green band emission in $\mathrm{ZnO}$ are merely recombination centres that are located at about $1 \mathrm{eV}$ from the edge of the valence band whereas the defect levels responsible for transient photoconductivity behaviour observed in our experiments are traps that do not participate in the recombination process and only capture and emit the holes. There may be a range of defect levels created at these energies in the material during the growth which depending on their charge state can act as recombination centres or traps. Variations in $s$ (the attempt to escape frequency) assumed for these calculations may explain the difference between the trap energies indicated by PL and extraction from photoconductivity measurements, where a value of $s=1 \times$ $10^{13} \mathrm{~Hz}$ would result in a trap energy centred at $0.92 \mathrm{eV}$ versus the value of $0.8 \mathrm{eV}$ obtained for $s=1 \times 10^{11} \mathrm{~Hz}$. The use of an accurate value for an attempt to escape frequency, requiring the measurement of the capture cross section for the hole traps, will improve the ability to resolve the trap energy spectrum.

\section{Conclusion}

In conclusion, the transient response of $\mathrm{ZnO}$ to visible excitation with energies below the bandgap energy was studied. A rate equation model to describe the observed behaviour is presented, and provides a means of extracting defect energy spectra given the assumptions of carrier capture and recombination lifetimes that are supported by TRPL data. Photoconductive response is found to be dominated by deep surface traps for unpassivated samples, and a lower density of shallower traps for samples passivated with $\mathrm{SiO}_{2}$. The observed trap energy spectrum peaked near $0.9 \mathrm{eV}$ in unpassivated samples and near $0.75 \mathrm{eV}$ in passivated samples. The responsible defect level for the green band defect level emission in $\mathrm{ZnO}$, which is at an energy close to the energy spectrum extracted for the hole traps responsible for persistent photoconductivity, has been previously attributed to different types of defects such as antisite oxide, $\mathrm{O}_{\mathrm{Zn}}[7,12]$, $\mathrm{Zn}$ vacancy, $V_{Z n}$ [8] and even oxygen vacancy, $V_{O}$ [14]. Further studies are needed to accurately identify the nature of the trap levels responsible for slow photoconductive transients and their relationship to defect levels responsible for the green band emission in $\mathrm{ZnO}$.

\section{Acknowledgments}

This work was supported by AFOSR under contract number FA9550-04-1-0390 and the Center for Optoelectronic Nanostructured Semiconductor Technologies, a DARPA UPR award HR0011-04-1-0040.

\section{References}

[1] Sharma P, Sreenivas K and Rao K V 2003 J. Appl. Phys. $933963-70$

[2] Jimenez Gonzalez A E and Soto Urueta J A 1998 Sol. Energy Mater. Sol. Cells 52 345-53

[3] Liu M and Kim H K 2004 Appl. Phys. Lett. 84 173-5

[4] Studenikin S A, Golego N and Cocivera M 1998 J. Appl. Phys. 84 5001-4

[5] Studenikin S A, Golego N and Cocivera M 2000 J. Appl. Phys. 87 2413-21

[6] Chung S J, Cha O H, Kim Y S, Hong C-H, Lee H J, Jeong M S, White J O and Suh E.-K 2001 J. Appl. Phys. 895454

[7] Lin B 2001 J. Electrochem. Soc. 148 G110-3

[8] Reynolds D C, Look D C, Jogai B, Van Nostrand J E, Jones R and Jenny J 1998 Solid State Commun. 106 701-4

[9] Reynolds D C, Look D C and Jogai B 2001 J. Appl. Phys. 896189

[10] Kang H S, Kang J S, Kim J W and Lee S Y 2004 J. Appl. Phys. 95 1246-50

[11] Ye J D et al 2005 Appl. Phys. A 81 759-62

[12] Hur T B, Jeen G S, Hwang Y H and Kim H K 2003 J. Appl. Phys. 94 5787-90

[13] Gaspar C, Costa F and Monteiro T 2001 J. Mater. Sci., Mater. Electron. 12 269-71

[14] Van Dijken A, Meulenkamp A E, Vanmaekelbergh D and Mijerink A 2000 J. Lumin. 87-89 454-6 
[15] Naito H, Nagase T, Ishii T, Okuda M, Kawaguchi T and Maruno S 1996 J. Non-Cryst. Solids 198-200 363-6

[16] Murphy T E, Chen D Y and Phillips J D 2005 Growth and electronic properties of $\mathrm{ZnO}$ epilayers by plasma-assisted molecular beam epitaxy J. Electron. Mater. 34 699-703

[17] Studenikin S A and Cocivera M 2002 J. Appl. Phys. 91 5060-5

[18] Murphy T E, Chen D Y, Cagin E and Phillips J D 2005 Electronic properties of $\mathrm{ZnO}$ epilayers grown on C-plane sapphire by plasma-assisted molecular beam epitaxy J. Vac. Sci. Technol. B 23 1277-80

[19] Murphy T E, Blaszczak J O, Moazzami K, Bowen W E and Phillips J D 2005 Properties of electrical contacts on bulk and epitaxial n-type $\mathrm{ZnO}$ J. Electron. Mater. 34 389-94

[20] Shimizu A, Kanbara M, Hada M and Kasuga M 1978 Japan. J. Appl. Phys. 171435
[21] Muret P, Philippe A, Monroy E, Munoz E, Beaumont B, Omnes F and Gibart P 2002 J. Appl. Phys. 91 2998-3001

[22] Nagai H, Zhu Q S, Kawaguchi Y, Hiramatsu K and Sawaki N 1998 Appl. Phys. Lett. 73 2024-6

[23] Hierro A, Kwon D, Ringel S A, Hansen M, Speck J S, Mishra U K and DenBaars S P 2000 Appl. Phys. Lett. $763064-6$

[24] Polyakov A Y, Smirnov N B, Usikov A S, Govorkov A V and Pushniy B V 1998 Solid-State Electron. 42 1959-67

[25] Polyakov A Y, Smirnov N B, Govorkov A V, Shin M, Skowronski M and Greve D W 1998 J. Appl. Phys. 84 870-6

[26] Auret F D, Meyer W E, Wu L, Hayes M, Legodi M J, Beaumont B and Gibart P 2004 Phys. Status Solidi $2012271-6$

[27] Salis M, Anedda A, Quarati F, Blue A J and Cunningham W 2005 J. Appl. Phys. 97 033709-1-6 